\title{
Electron flux enhancement in the inner radiation belt during moderate magnetic storms
}

\author{
H. Tadokoro ${ }^{1}$, F. Tsuchiya ${ }^{1}$, Y. Miyoshi ${ }^{2}$, H. Misawa ${ }^{1}$, A. Morioka ${ }^{1}$, and D. S. Evans ${ }^{3}$ \\ ${ }^{1}$ Planetary Plasma and Atmospheric Research Center, Tohoku University, Aoba-ku, Sendai, 980-8578, Japan \\ ${ }^{2}$ Solar-Terrestrial Environment Laboratory, Nagoya University, Nagoya, 464-8601, Japan \\ ${ }^{3}$ Space Environment Center, NOAA, Boulder, CO 80305, USA
}

Received: 26 September 2006 - Revised: 3 May 2007 - Accepted: 21 May 2007 - Published: 29 June 2007

\begin{abstract}
During moderate magnetic storms, an electron channel $(300-1100 \mathrm{keV})$ of the NOAA satellite has shown sudden electron flux enhancements in the inner radiation belt. After examinating the possibility of contamination by different energetic particles, we conclude that these electron flux enhancements are reliable enough to be considered as natural phenomena, at least for the cases of small to moderate magnetic storms. Here, we define small and moderate storms to be those in which the minimum $D_{s t}$ ranges between -30 and $-100 \mathrm{nT}$. The electron flux enhancements appear with over one order of magnitude at $\mathrm{L} \sim 2$ during these storms. The enhancement is not accompanied by any transport of electron flux from the outer belt. Statistical analysis shows that these phenomena have a duration of approximately 1 day during the period, starting with the main phase to the early recovery phase of the storms. The flux enhancement shows a dawndusk asymmetry; the amount of increased flux is larger in the dusk side. We suggest that this phenomenon could not be caused by the radial diffusion but would be due to pitch-angle scattering at the magnetic equator. The inner belt is not in a stationary state, as was previously believed, but is variable in response to the magnetic activity.
\end{abstract}

Keywords. Magnetospheric physics (Energetic particles, precipitating; Energetic particles, trapped; Storms and substorms)

\section{Introduction}

The variability of energetic particles in the Earth's radiation belts has been studied in order to understand particle acceleration and loss processes and their relationship to magnetospheric disturbances (see the review for electron radiation belts by Friedel et al., 2002, and the references therein). It

Correspondence to: $\mathrm{H}$. Tadokoro

(syougun@pparc.geophys.tohoku.ac.jp) is also important from the perspective of space weather to realize and predict the flux variation of the trapped particles.

Several processes for electron acceleration have been proposed to account for the dynamical flux variations in accordance with magnetospheric disturbances. Radial diffusion has been discussed from the standpoint of an external source (e.g. Schultz and Lanzerotti, 1974). Some mechanisms pertaining to the internal source mechanisms have also been discussed. In particular, the wave-particle interaction process is regarded as a plausible acceleration process during the geomagnetically disturbed periods (e.g. Meredith et al., 2002; Miyoshi et al., 2003, 2007; Horne et al., 2005).

Compared to the outer radiation belt, the inner belt has been believed to be a region where energetic electrons exhibit little temporal variability, except when an extremely huge magnetic storm impacts the distribution of the inner belt electrons (e.g. Gusev et al., 1995; Baker et al., 2004). Only a few reports have been presented that demonstrate the response of high energy electrons in the inner region to a magnetic storm (Kikuchi and Evans, 1989; Morioka et al., 2001; Miyoshi et al., 2002). By using data from the NOAA satellite, Kikuchi and Evans (1989) showed that a $>30-\mathrm{keV}$ electron flux at the lower $\mathrm{L}(\sim 1.3)$ exhibited a sudden enhancement during a magnetic storm. Morioka et al. (2001) and Miyoshi et al. (2002) also reported that a $300-\mathrm{keV}$ electron flux enhancement in the inner belt $(\mathrm{L} \sim 2)$ was synchronized with the onset of the outer belt electron decrease during some magnetic storms.

A polar orbiting low-altitude satellite such as NOAA is appropriate for observing flux distributions and their dynamics over a wide range of $\mathrm{L}$ with high time resolution because it permits a detailed comparison between the outer and inner radiation belts within time scales of the development of storms. In this paper, we investigate the variability of the electron flux in the inner belt and its relation to magnetic storms.

Published by Copernicus Publications on behalf of the European Geosciences Union. 


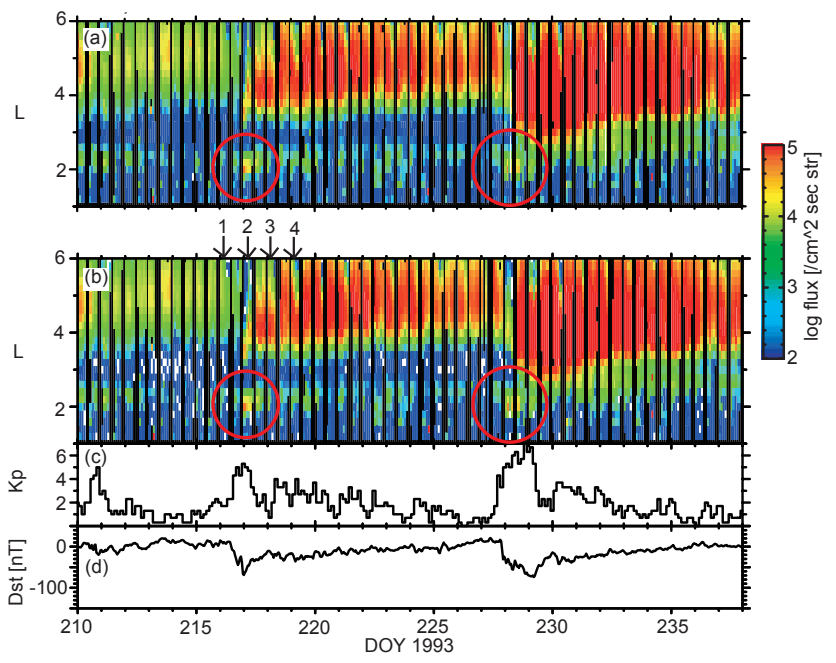

Fig. 1. L-t diagram for a $300-\mathrm{keV}$ electron flux derived from the $90^{\circ}$ sensor (a), and after subtraction of the count rates of the $0^{\circ}$ sensor from that of the $90^{\circ}$ sensor (b). (c) The $K_{p}$ index. (d) The $D_{s t}$ index.

\section{Observations}

To investigate variations of energetic particle flux in the inner belt, we used data from the NOAA 10 and NOAA 12 satellites that were in a Sun-synchronous polar orbit with an inclination of $98^{\circ}$ and an altitude of about $800 \mathrm{~km}$. The orbital period was about $102 \mathrm{~min}$. The local times of the ascending and descending phases of the satellites were $19.5 \mathrm{~h}$ (dusk side) and $7.5 \mathrm{~h}$ (dawn side), respectively. The Medium Energy Proton Electron Detector (MEPED) on board the NOAA satellites measured the electron counts in three energy channels (E1: $30-1100$, E2: 100-1100, and E3: $300-1100 \mathrm{keV}$ ), proton counts in five energy channels (P1: 30-80, P2: 80-250, P3: 250-800, P4: 800-2500, and P5: >2500 keV), and one omnidirectional channel (P6: $>16 \mathrm{MeV})$. It is well known that the P6 channel also detects $>1 \mathrm{MeV}$ electrons (Miyoshi et al., 2004). The MEPED is made up of two sensors; one called the $0^{\circ}$ sensor, which views radially outward along the vector from the Earth to the satellite, and the other called the $90^{\circ}$ sensor, which views in a direction perpendicular to the $0^{\circ}$ sensor. The details of the MEPED are described in Raben et al. (1995). In the present study, we made use of the data during the period from 1989 to 1998. Taking into account the flux variation caused by the offset of the terrestrial magnetic field, we restricted the data to a geographic longitude ranging from $180^{\circ}$ to $280^{\circ}$ in the Northern Hemisphere, and from $180^{\circ}$ to $250^{\circ}$ in the Southern Hemisphere. The electrons of the $90^{\circ}$ sensor are in the drift loss cone (Blake et al., 2001).

\section{Results and discussion}

\subsection{The inner electron flux enhancement event}

Figure 1a shows the 2-h-averaged flux of energetic electrons in the channel of $300-1100 \mathrm{keV}$, plotted in the L-t diagram, during the period from the 210 to 238 days of 1993. Figures $1 \mathrm{c}$ and d present the $K_{p}$ and $D_{s t}$ indices, respectively. There were two moderate magnetic storms during this period, whose minimum $D_{s t}$ indices were greater than $-100 \mathrm{nT}$.

In Fig. 1, the electron channel showed rapid flux enhancements in the inner belt $(2<\mathrm{L}<2.5)$ during the main phase of the magnetic storms (DOY 217 and 228). The most significant point is that the electron flux at $\mathrm{L}=2$ (the inner belt) increased by more than one order of magnitude during the main phase before the flux increase in the outer belt. The peak of the inner zone depends on data selection. An electron flux peak $\mathrm{L}<2$ (Lyons et al., 1972) is observed in the South Atlantic Anomaly (SAA), where many particles can precipitate deeply into the atmosphere due to a lower mirror point. However, we did not select this region, and restricted the data for geographic longitude ranging from $180^{\circ}$ to $280^{\circ}$ in the Northern Hemisphere and from $180^{\circ}$ to $250^{\circ}$ in the Southern Hemisphere. Therefore, the peak of the inner belt can be seen around $\mathrm{L}=2$ in the present data set. The duration of the inner belt enhancement was about 1-1.5 days. It should be noted that the inner belt enhancement was separated from the outer belt by the distinct slot region. These inner belt flux enhancements were mostly found during moderate intensity storms. Although intense enhancements also occurred during great storms $(<-100 \mathrm{nT})$, it is difficult to distinguish the flux enhancement in the inner belt from the violent injection from the outer belt. Hence, we concentrated only on moderate storms in this study.

\subsection{Examination of the possibility of contamination}

The example shown in Fig. 1a has clearly demonstrated the flux enhancement of energetic electrons in the inner radiation belt during moderate magnetic storms. Before investigations of this phenomena, it should be examined whether the detected flux enhancement in the E3 channel of MEPED really does result from electrons of $300-1100 \mathrm{keV}$ or is it a result of an apparent enhancement due to the contamination by different energetic particles. The E3 (300-1100 keV) channel has some response to energetic protons having energies of 440 $2700 \mathrm{keV}$ and $>90 \mathrm{MeV}$, and to electrons having energies of $>6 \mathrm{MeV}$ (Evans and Greer, 2000). In addition to these possible sources of contamination, it should also be mentioned that the count rate of the E3 channel may become uncertain when the P1 $(30-80 \mathrm{keV})$ channel has count rates that are similar to the E3 channel.

Figure 2 shows the variation in the count rate (top) and the $\mathrm{L}$ value (bottom) corresponding to the DOY 217 storm in 


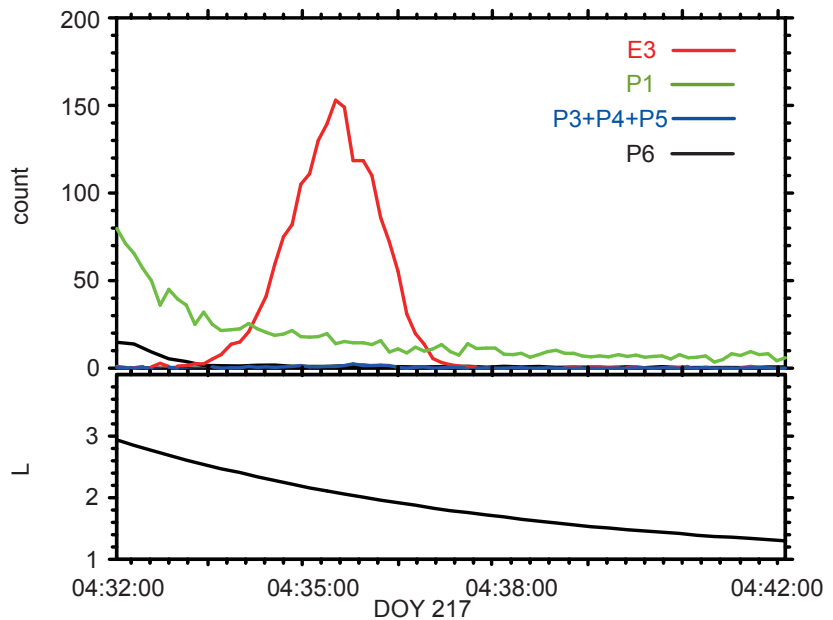

Fig. 2. Variation of the count rates at various channels at the DOY 217 storm. The red, green, blue, and black lines show the count rates of the E3, P1, P3+P4+P5, and P6 channels, respectively. The bottom panel indicates the variation of $\mathrm{L}$.

Fig. 1. The red, green, blue, and black lines indicate count rates of $\mathrm{E} 3, \mathrm{P} 1, \mathrm{P} 3+\mathrm{P} 4+\mathrm{P} 5$, and $\mathrm{P} 6$, respectively. It can be seen that the $\mathrm{E} 3$ count rates increased around $\mathrm{L}=2$. At the same time, the count rates obtained from $\mathrm{P} 1$ were found to be about one-tenth. In addition, the trend in variations of E3 and P1 was completely different. Thus, we conclude that proton flux from P1 of $30-80 \mathrm{keV}$ did not contribute to the corresponding flux enhancement in E3 for this case. Regarding the possibility of proton contamination (440-2700 keV), we can examine the possibility by estimating the sum of the P3, P4, and P5 count rates. The count rates were virtually zero during the enhancement of E3. Therefore, we conclude that the $300-\mathrm{keV}$ electron flux enhancement is not due to the contamination from protons (440-2700 keV). Finally, the variation of the P6 count rates provides a rough estimation of the possible contamination by $>90 \mathrm{MeV}$ protons and $>6 \mathrm{MeV}$ electrons (Evans and Greer, 2000). It can be seen that the count rates of the $\mathrm{P} 6$ channel were almost zero around $\mathrm{L}=2$. Thus, we can exclude the possibility of contamination by $>90 \mathrm{MeV}$ protons and $>6 \mathrm{MeV}$ electrons.

Besides the above discussion, we defined the precipitating electron flux by subtracting counts of the $0^{\circ}$ sensor from those of the $90^{\circ}$ sensor. Figure $1 \mathrm{~b}$ shows the variation of electron flux $(300 \mathrm{keV})$ extracted the $0^{\circ}$ sensor from the $90^{\circ}$ sensor. The white color indicates the area where the electron flux from the $0^{\circ}$ sensor was greater than that from the $90^{\circ}$ sensor. Assuming a uniform angular distribution of contamination, it is natural to consider that the contamination of the $90^{\circ}$ sensor should be equal to that of the $0^{\circ}$ sensor. If the E3 channel contains the contamination, the $90^{\circ}$ and $0^{\circ}$ sensors comprise true count rates from each sensor and equal contamination, respectively. Thus, the electron flux in Fig. 1b underestimates electron flux compared to the true electron

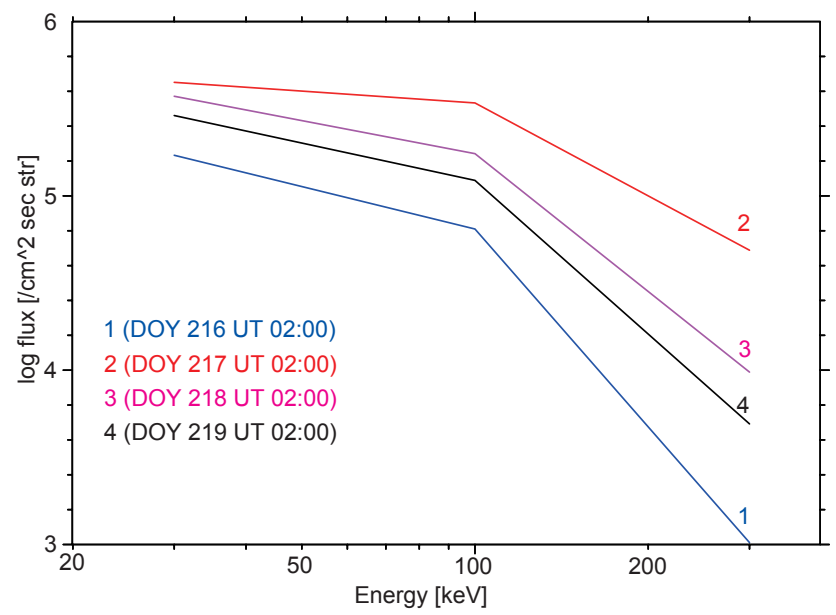

Fig. 3. Electron energy spectrum during the initial phase (spectrum 1), the main phase (spectrum 2), and the recovery phase (spectrum 3,4$)$ of the DOY 217 storm.

flux. However, it can be seen that the electron flux enhancement appears in the inner belt during the two storms. This is one of the results which supports that the electron flux enhancement is not due to contamination.

From these examinations, we conclude that the present phenomena of the inner electron flux enhancement, as shown in Fig. 1, are due to the 300-1100 keV electrons. Hereafter, we examine the data sets in which the count rate of the $0^{\circ}$ sensor data have been subtracted from that of the $90^{\circ}$ sensor, as shown in Fig. 1b.

\subsection{Characteristic of the inner electron flux enhancement}

To investigate variations in the electron spectral shape in response to magnetic storms, we examined the variability in electron energy spectra at $\mathrm{L}=2$ during these moderate magnetic storms. Figure 3 shows energy spectra on the DOY 217 , where the vertical axis represents the logarithm of the flux and the horizontal axis, the particle energy. The numbers alongside the figures correspond to the time shown by the arrows in Fig. 1b. The spectrum changed from soft to hard during the main phase of the storm (from Spectrum 1 to 2), corresponding to the electron flux enhancement, as shown in Fig. 1. After this enhancement, the spectra in the storm recovery phase (Spectrum 3,4) turned again to soft, as in the initial phase (Spectrum 1). This suggests that the higher energy component of the electron flux in the inner belt increased more than the lower energy component during the main phase.

The statistical behavior of the electron flux enhancement in the inner belt during small and moderate magnetic storms was studied by means of the superposed epoch analysis. We selected 32 moderate storms that involved electron flux enhancement events in the inner belt during the period from 1989 to 1998 . We used the data from the NOAA 10 and 


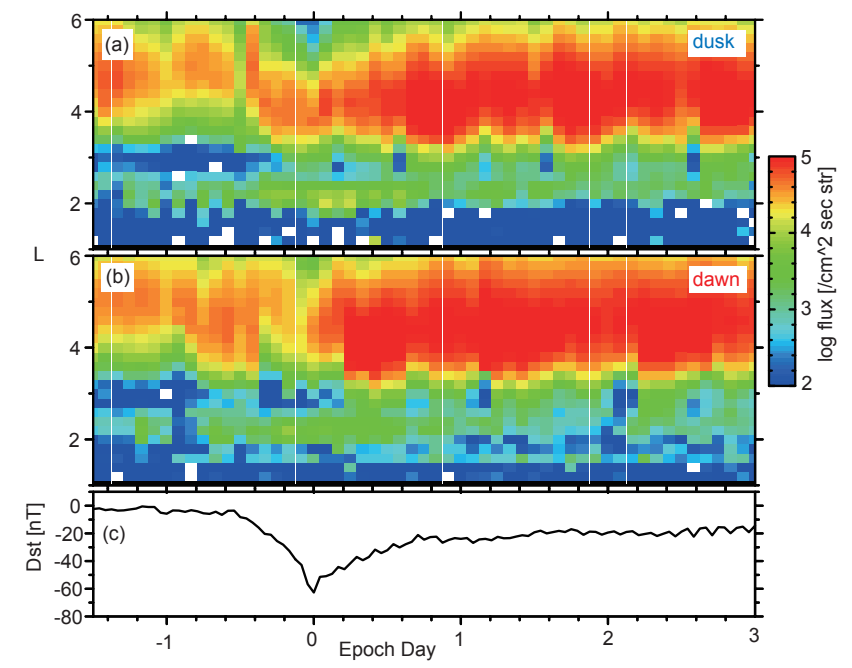

Fig. 4. Superposed electron flux (300-1100 keV), and $D_{s t}$ index for 32 storms. The zero epoch is taken to be the time of the minimum $D_{s t}$ value. (a) 2-h-averaged electron flux at the dusk side. (b) 2h-averaged electron flux at the dawn side. (c) the superposed $D_{s t}$ index.

NOAA 12 satellites during the period from 1989 to 1991 and from 1992 to 1998. Here, we define small and moderate storms to be those in which the minimum $D_{s t}$ ranged between -100 and $-30 \mathrm{nT}$. The zero epoch is taken to be the time of the minimum $D_{s t}$ value. The result of the analysis is shown in Fig. 4, where Figs. 4a and b show L-t diagrams of the superposed 2-h-averaged flux variation from the $90^{\circ}$ sensor at the dusk and dawn sides, respectively. From these figures it can be seen that inner belt electrons around $\mathrm{L} \sim 2.2$ increase during the main phase ( $\sim-0.4$ day) by about one order of magnitude, whereas the flux of the outer belt decreases significantly. The duration of the enhancement was found to be about 1 day from this analysis. The analysis also showed a local time dependence: The enhancement in the flux at the dusk side was found to be greater than that at the dawn side. This local time asymmetry suggests that the phenomena are related to local processes. Our statistical study also indicated that the magnitude of the flux enhancement did not correlate with the minimum $D_{s t}$ (not shown).

Another interesting point to note here is that the flux enhancement was observed at lower $\mathrm{L}$ values than for the typical inner belt positions observed by NOAA. A typical example shown in Fig. 5 illustrates the electron flux variation from the 157 to 163 days of year 1992. The inner belt is faint but can be steadily observed before the storm in the region from $\mathrm{L}=2$ to $\mathrm{L}=2.5$. Just as the main phase of the storm begins on DOY 308, the inner belt flux enhancement was observed at a region having a lower $\mathrm{L}(\mathrm{L} \sim 2)$ value than usual. The same feature is also confirmed in Fig. 1.

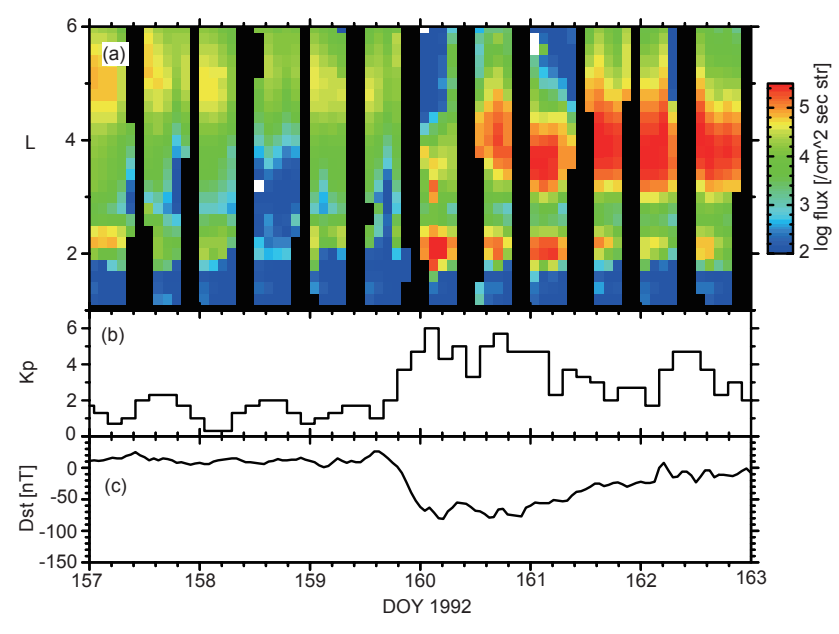

Fig. 5. (a) L-t diagram of electron flux variations in the radiation belt from the 157 to 163 days of 1992. (b) The $K_{p}$ index. (c) The $D_{s t}$ index.

3.4 Possible mechanisms for the enhancement of inner belt electrons

One of possible mechanism explaining the inner belt flux enhancement during a magnetic storm is radial diffusion $(\mathrm{Gu}-$ sev et al., 1995), a process by which particles diffuse from a region of higher phase-space density region to a lower one. The electron flux enhancement event investigated in this study, however, did not exhibit a corresponding transport from the outer belt to the inner belt across the slot region, as shown in Fig. 1, and Fig. 4. Loto' aniu et al. (2006) estimated the time scale of radial diffusion for energetic electrons for the case of the 2003 Halloween storm. Their results were consistent with the diffusion time scales of $12 \mathrm{~h}$ over $\mathrm{L}=2.1-3.3$ observed by SAMPEX during the storm. The diffusion time scale for the case of small to moderate storms mentioned in the present study would be longer compared to the great storm because the radial diffusion coefficients depend on magnetic activity (e.g. Brautigam and Albert, 2000). However, the inner electron flux enhancement event occurred within $12 \mathrm{~h}$ from the start (beginning of the main phase) of the small or moderate storms. Thus, we can conclude that this electron flux enhancement is not caused by the radial diffusion process from the outer belt.

Another possible mechanism for the present phenomenon would be pitch-angle scattering of the trapped electrons by wave-particle interactions at the magnetic equator. The evidence of precipitation for energetic electrons $(\sim$ several tens of $\mathrm{keV}$ ) are observed at low altitude (e.g. Inan et al., 1984). Imhof et al. (1983) showed that such precipitations are caused by VLF signals during the quiet time. To examine this process, we should first investigate the existence of waves that can interact with the electrons during magnetic storms in the inner magnetosphere. Meredith et al. (2004) 
showed that plasmaspheric hiss emissions during substorm activity distribute mainly in the dayside. Enhanced plasmaspheric hiss or other waves could be a possible candidate for the cause of pitch-angle scattering in the inner belt. In the near future, we plan to clarify such a relationship between the generation of whistler mode waves and the response of the inner radiation belt electrons during magnetic storms.

\section{Summary}

We have examined electron (300-1100 keV) flux enhancements in the inner belt during small to moderate magnetic storms. Our results show that the NOAA observed electron flux enhancement in the inner belt is not due to the contamination from other energetic particles in small and moderate magnetic storms. The enhancement of electron flux also showed characteristics that were different from those in the outer belt. The results are summarized as follows:

1. The flux of inner belt electrons increases by over one order of magnitude during the main phase of magnetic storms. This is a contrary variation to the outer belt.

2. The duration of such electron flux enhancements is approximately 1 day.

3. During the event, the electron flux shows spectral hardening.

4. There is a local time dependence on the flux enhancement.

5. The flux enhancement appears at lower $L(\sim 2)$ values than the typical inner belt positions ( $\mathrm{L} \sim 2.2)$ observed by NOAA.

Acknowledgements. We thank K. T. Asai for useful discussions regarding problems related to contamination of the MEPED instrument. The $D_{s t}$ and $K_{p}$ indicies are provided from WDC-C2 in Kyoto University. NOAA through the WDC-C2 for Aurora, National Institute of Polar Research, Japan.

Topical Editor I. A. Daglis thanks R. Selesnick and another anonymous referee for their help in evaluating this paper.

\section{References}

Baker, D. N., Kanekal, S. G., Li, X., Monk, S. P., Goldstein, J., and Burch, J. L.: An extreme distortion of the Van Allen belt arising from the Hallowe'en solar storm in 2003, Nature, 432, 878-881, 2004.

Blake, J. B., Inan, U. S., Walt, M., Bell, T. F., Bortnik, J., Chenette, D. L., and Christian, H. J.: Lightning-induced energetic electron flux enhancements in the drift loss cone, J. Geophys. Res., 106, 29733-29744, 2001.

Brautigam, D. H. and Albert, J. M.: Radial diffusion analysis of outer radiation electrons during the October 9, 1990, magnetic storm, J. Geophys. Res., 105, 291-309, 2000.
Evans, D. S. and Greer, M. S.: Polar Orbiting Environmental Satellite Space Environment Monitor - 2: Instrument Descriptions and Archive Data Documentation, Space Environment Center, Boulder, Colorado, 2000.

Friedel, R. H. W., Reeves, G. D., and Obara, T.: Relativistic electron dynamics in the inner magnetosphere-a review, J. Atmos. Sol. Terr. Phys., 64, 265-282, 2002.

Gusev, A. A., Kohno, T., Martin, I. M., Pugacheva, G. I., Turtelli Jr., A., Tylka, A. J., and Kudela, K.: Injection and fast radial diffusion of energetic electrons into the inner magnetosphere, Planet. Space Sci., 43, 1131-1134, 1995.

Horne, R. B., Thorne, R. M., Shprits, Y. Y., Meredith, N. P., Glauert, S. A., Smith, A. J., Kanekal, S. G., Baker, D. N., Engebretson, M J., Posch, J. L., Spasojevic, M., Inan, U. S., Pickett, J. S., and Decreau, P. M. M.: Wave acceleration of electrons in the Van Allen radiation belts, Nature, 437, 227-230, doi:10.1039/nature03939, 2005.

Imhof, W., Reagan, J. B., Voss, H. D., Gaines, E. E., Datlowe, D. W., and Mobilia, J.: DIRECT Observation of radiation belt electrons precipitated by the controlled injection VLF signals from ground-based transmitter, Geophys. Res. Lett., 10, 361364, 1983.

Inan, U. S., Chang, H. C., and Helliwell, R. A.: Electron Precipitation Zones Around Major Ground-Based VLF Signal Sources, J. Geophys. Res., 89, 2891-2906, 1984.

Kikuchi, T. and Evans, D. S.: Energetic electrons observed by NOAA-6 over Japan $(\mathrm{L}=1.3)$ at the time of geomagnetic storm on February 8-9, Proc. Res. Inst. Atmos., Nagoya University, 36, 137-149, 1989.

Loto'aniu, T. M., Mann, I. R., Ozeki, L. G., Chan, A. A., Dent, Z. C., and Milling, D. K.: Radial diffusion of relativistic electrons into the radiation belt slot region during the $2003 \mathrm{Hal}-$ loween geomagnetic storms, J. Geophys. Res., 111, A04218, doi:10.1029/2005JA011355, 2006.

Lyons, L. R., Thorne, R. M., and Kennel, C. F.: Pitch-Angle Diffusion of Radiation Belt Electrons within the Plasmasphere, J. Geophys. Res., 77, 3455-3474, 1972.

Meredith, N. P., Horne, R. B., Summers, D., Thorne, R. M., Iles, R. H. A., Heynderickx, D., and Anderson, R. R.: Evidence for acceleration of outer zone electrons to relativistic energies by whistler mode chorus, Ann. Geophys., 20, 967-979, 2002, http://www.ann-geophys.net/20/967/2002/.

Meredith, N. P., Horne, R. B., Thorne, R. M., Summers, D., and Anderson, R. R., Substorm dependence of plasmaspheric hiss, J. Geophys. Res., 109, A06209, doi:10.1029/2004JA010387, 2004.

Miyoshi, Y., Morioka, A., Obara, T., and Nagai, T.: Dynamics of the energetic particles during the storm main phase - EXOS-D (Akebono) and NOAA observations -, Eos. Trans. AGU, 83(22), West. Pac. Geophys. Meet. Suppl., Abstract SP21A-01, 2002.

Miyoshi, Y., Morioka, A., Obara, T., Misawa, H., Nagai, T., and Kasahara, Y.: Rebuilding process of the outer radiation belt during the 3 November 1993 magnetic storm: NOAA and Exos-D observations, J. Geophys. Res., 108(A1), 1004, doi:10.1029/2001JA007542, 2003.

Miyoshi, Y. S., Jordanova, V. K., Morioka, A., and Evans, D. S.: Solar cycle variations of the electron radiation belts: Observations and radial diffusion simulation, Space Weather., 2, S10S02, doi:10.1029/2004SW000070, 2004.

Miyoshi, Y., Morioka, A., Kataoka, R., Kasahara, Y., and Mukai, T.: 
Evolution of the outer radiation belt during the November 1993 storms driven by corotating interaction regions, J. Geophys. Res., 112, A05210, doi:10.1029/2006JA012148, 2007.

Morioka, A., Miyoshi, Y., and Obara, T.: Dynamics of Energetic Particles in the Inner Radiation Belt During Magnetic Storms, International Space Environment Conference 2001, Queenstown, New Zealand, 2001.
Raben, V. J., Evans, D. S., Sauer, H. H., Sahm, S. R., and Huynh, M.: TIROS/NOAA satellite space environment monitor data archive documentation: 1995 update, NOAA Tech. Memo. ERL SEL-86, 1995.

Schultz, M. and Lanzerotti, L. J.: Particle diffusion in the radiation belts, in: Physics and Chemistry in Space, Springer-Verlag, Berlin, 1974. 